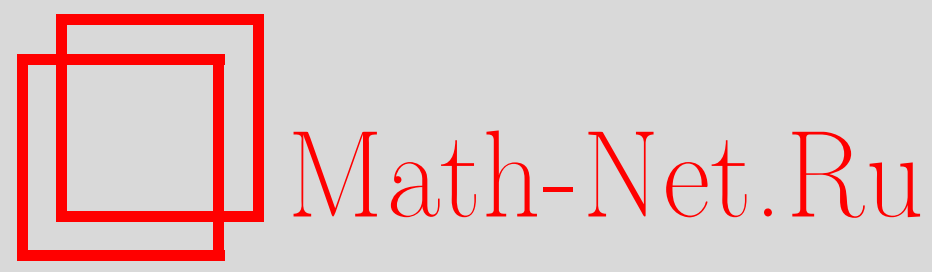

Д. С. Малышев, О пересечении и симметрической разности семейств граничных классов для задач о раскраске и о хроматическом числе, Дискрет. матем., 2012, том 24, выпуск $2,75-78$

DOI: https://doi.org/10.4213/dm1184

Использование Общероссийского математического портала Math-Net.Ru подразумевает, что вы прочитали и согласны с пользовательским соглашением http://www.mathnet.ru/rus/agreement

Параметры загрузки:

IP: 54.205 .225 .156

26 апреля 2023 г., 03:01:27 


\title{
О пересечении и симметрической разности семейств граничных классов для задач о раскраске и о хроматическом числе
}

\author{
() 2012 г. Д. С. Малышев
}

\begin{abstract}
В работе исследуются семейства граничных классов для задач о вершинной $k$-раскраске и о хроматическом числе. Указано континуальное семейство классов графов, являющихся граничными одновременно для первой задачи при $k=3$ и для второй. Для любого $k>3$ выявлено континуальное семейство граничных классов для первой задачи, не являющихся граничными для второй. Для задачи о хроматическом числе найден граничный класс, не являющийся граничным для задачи о вершинной $k$-раскраске ни при каком $k$.
\end{abstract}

\section{1. Введение}

Данная работа является продолжением статей $[1,2]$, в которых для задач о раскраске исследовалась граница между «простыми» и «сложными» классами в решетке наследственных классов, то есть классов, замкнутых относительно удаления вершин. Каждый наследственный (и только наследственный) класс $\mathscr{X}$ определяется множеством своих запрещенных порожденных подграфов $\mathscr{Y}$, в этом случае принята запись $\mathscr{X}=\operatorname{Free}(\mathscr{y})$. Хорошо известно, что минимальное по включению множество У с таким свойством существует, единственно и обозначается через Forb $(\mathscr{X})$. Если Forb( $\mathscr{X})$ конечно, то $\mathscr{X}$ называется конечно определенным.

Примерами конечно определенных классов являются такие классы, как множество всех графов, у которых ограничена сверху наибольшая степень вершин, или класс реберных графов. Вместе с тем, классы планарных и двудольных графов таким свойством не обладают.

Формализуем понятия «простого» и «сложного» класса. Пусть П - какая-либо $N P$-полная задача на графах. Наследственный класс называется П-простым, если задача П для графов из этого класса полиномиально разрешима, и П-сложным в противном случае. На протяжении данной работы предполагается, что $P \neq N P$, и это условие не включается явно в формулировки соответствующих утверждений.

Подход к выявлению границы, рассматриваемый в работах указанного цикла, основан на понятии граничного класса графов, введенного в [3] и уточненного в [4]. Значение этого понятия состоит в том, что любой конечно определенный класс графов является П-сложным тогда и только тогда, когда он включает некоторый П-граничный класс. 
Класс $\mathscr{X}$ называется П-предельным, если существует такая бесконечная монотонно убывающая последовательность $\mathscr{X}_{1} \supseteq \mathscr{X}_{2} \supseteq \ldots$ из П-сложных классов, что $\mathscr{X}=\bigcap_{i=1}^{\infty} \mathscr{\mathscr { X }} i$. Минимальный по включению П-предельный класс называется П-граничным.

В данной работе рассматриваются две задачи на графах: задача о вершинной $k$-раскраске (задача $k$-ВР) и задача о хроматическом числе (задача ХЧ). Напомним, что в первой из них для заданного графа $G$ требуется определить, меньше ли хроматическое число графа $G$ фиксированного натурального числа $k$. В задаче ХЧ заданы два параметра - граф $G$ и натуральное число $s$, для которых требуется определить, меньше ли хроматическое число $G$ числа $s$. Отметим, что ХЧ-простой класс - наследственный класс графов, для которого существует полиномиальный алгоритм проверки выполнения соответствующего неравенства, пригодный сразу для всех значений $s$.

Цель настоящей работы - изучение семейств граничных классов для задач $k$-ВР и ХЧ. В статье указывается континуальное семейство классов графов, являющихся одновременно 3-ВР-граничными и ХЧ-граничными. Конструктивным образом показывается, что при любом $k>3$ существует континуальное множество $k$-ВР-граничных классов, ни один из которых не является ХЧ-граничным. Выявлен ХЧ-граничный класс, при любом $k$ не являющийся $k$-ВР-граничным.

В работе приняты следующие обозначения и определения: $\chi(G)$ - хроматическое число графа $G ; G_{1} \circ G_{2}$ - результат умножения графов $G_{1}=\left(V_{1}, E_{1}\right)$ и $G_{2}=\left(V_{2}, E_{2}\right)$, то есть граф $G=\left(V_{1} \cup V_{2}, E_{1} \cup E_{2} \cup V_{1} \times V_{2}\right)$; $\mathscr{D}$ - множество графов, являющихся реберными к графам, каждая компонента связности которых является деревом с не более чем тремя листьями; со(D) - множество дополнительных графов к графам из $\mathscr{D}$; Colour $(k)=\{G: \chi(G) \leqslant k\} ; \mathscr{L}^{p}, p \in\{0,1,2, \ldots\},-$ множество порожденных подграфов графов множества $\left\{G \circ K_{p}, G \in \mathscr{X}\right\}, G \circ K_{0}=G ; \mathcal{B}_{k}$ - множество $k$-ВР-граничных классов; $\mathcal{B}-$ множество ХЧ-граничных классов.

\section{2. Вспомогательные результаты}

Лемма 1. Пусть $\mathscr{\Re}-$ П-предельный класс и существует такой конечно определенный класс $\mathscr{X}$, что $\mathscr{B} \subseteq \mathscr{X}$. Тогда для любого иелого неотрииательного числа $p$ класс $\mathscr{B}^{p}$ является пределом бесконечной монотонно убывающей последовательности П-сложных подклассов класса $\mathscr{L}^{p}$.

Доказательство. Сначала докажем утверждение леммы для случая $p=0$. Рассмотрим любую бесконечную монотонно убывающая последовательность $\left\{\mathscr{B}_{i}\right\}$ из П-сложных классов, сходящуюся к классу $\mathscr{B}$. Понятно, что для любого графа $G \in \operatorname{Forb}(\mathscr{X})$ существует такое натуральное число $i_{G}$, что для любого $i>i_{G}$ справедливо включение $\mathscr{B}_{i} \subseteq \operatorname{Free}(\{G\})$. Пусть $i^{*}-$ наибольшее из чисел множества $\left\{i_{G}: G \in \operatorname{Forb}(\mathscr{C})\right\}$. Ясно, что члены последовательности $\left\{\mathscr{P}_{i}\right\}$, начиная с номера $i *$, образуют сходящуюся к $\mathscr{B}$ бесконечную монотонно убывающую последовательность П-сложных частей класса $\mathscr{X}$.

Для доказательства леммы при $p \neq 0$ заметим, что $\mathscr{乃}^{p}-$ предел последовательности $\left\{\mathscr{P}_{i}^{p}\right\}$, все члены которой, начиная с $i^{*}$-ого, являются подклассами $\mathscr{L}^{p}$. Лемма 1 доказана.

Лемма 2. Если $\mathscr{B} \in \mathcal{B}_{3}$ и для некоторого конечно определенного класса $\mathscr{\mathscr { L }}$ справедливо включение $\mathscr{B} \subseteq \mathscr{L} \subseteq$ Colour(4), то $\mathscr{B} \in \mathcal{B}$.

Доказательство. Поскольку при любом $k$ в любом классе графов задача $k$-ВР полиномиально сводима к задаче ХЧ, то класс $\mathscr{B}-\mathrm{XЧ-предельный.} \mathrm{Покажем,} \mathrm{что} \mathrm{этот} \mathrm{класс}$ является ХЧ-граничным. 
Предположим противное, тогда существует такой ХЧ-граничный класс, что $\mathscr{B}^{\prime} \subset \mathscr{B}$. Из доказательства леммы 1 следует, что в любой бесконечной монотонно убывающей последовательности ХЧ-сложных классов, сходящейся к классу $\mathscr{R}^{\prime}$, имеется элемент, начиная с которого все классы - подклассы $\mathscr{X}$. Так как $\mathscr{X} \subseteq \operatorname{Colour}(4)$, то для любого $k \geqslant 4$ класс $\mathscr{X}$ является $k$-ВР-простым. Поскольку в классе всех графов задачи 1-ВР и 2-ВР полиномиально разрешимы, то в классе $\mathscr{X}$ задачи 3 -ВР и ХЧ полиномиально эквивалентны. Поэтому класс $\mathscr{B}^{\prime}-3$-ВР-предельный. Получаем противоречие с тем, что $\mathscr{B} \in \mathcal{B}_{3}$. Лемма 2 доказана.

Лемма 3. Для любого $\mathscr{B} \in \mathcal{B}_{k}$ справедливо включение $\mathscr{B} \subseteq \operatorname{Colour}(k)$.

Доказательство. Предположим, что существует граф $G \in \mathscr{B} \backslash$ Colour $(k)$. Пусть $\mathscr{X}$ есть некоторый $k$-ВР-сложный надкласс класса $\mathscr{B}$. Тогда $G \in \mathscr{X}$. Рассмотрим класс $\mathscr{X}^{\prime}=\mathscr{X} \cap \operatorname{Free}(\{G\})$. Покажем, что $\mathscr{X}^{\prime}$ является $k$-ВР-сложным.

Очевидно, что задача принадлежности любому из классов $\mathscr{X}^{\prime}$ и $\mathscr{X} \backslash \mathscr{X}^{\prime}$ для графов из $\mathscr{X}$ решается за полиномиальное время. Вместе с тем, хроматическое число любого графа из $\mathscr{L} \backslash \mathscr{L}^{\prime}$ не меньше $k+1$, поэтому задача $k$-ВР для графов из этого класса решается за время $O(1)$. Таким образом, задача $k$-ВР для графов из класса $\mathscr{X}$ полиномиально сводится к той же задаче для графов из $\mathscr{X}^{\prime}$. Поэтому $\mathscr{X}^{\prime}-k$-ВР-сложный граф.

Таким образом, сделанное предположение приводит к существованию бесконечной монотонно убывающая последовательность $k$-ВР-сложных подклассов класса Free $(\{G\})$, имеющей своим пределом класс $\mathscr{B}$. Но тогда $\mathscr{B} \subseteq \operatorname{Free}(\{G\})$, что влечет противоречие. Лемма 3 доказана.

\section{3. О некоторых граничных классах для задач о раскраске и о хроматическом числе}

Теорема 1. Пусть $\mathscr{B} \in \mathcal{B}_{k}, k \geqslant 3, u$ для некоторого конечно определенного класса $\mathscr{X}$ справедливо включение

$$
\mathscr{B} \subseteq \mathscr{X} \subseteq \operatorname{Colour}(k+1)
$$

Тогда для любого натурального р класс $\mathscr{B}^{p}$ принадлежит семейству $\mathcal{B}_{k+p}$.

Доказательство. Поскольку класс $\mathscr{B}$ является $k$-ВР-предельным, то существует такая последовательность $\mathscr{B}_{1} \supseteq \mathscr{B}_{2} \supseteq \ldots$, состоящая из $k$-ВР-сложных классов, что

$$
\mathscr{B}=\bigcap_{i=1}^{\infty} \mathscr{B}_{i}
$$

Покажем, что любой класс последовательности $\left\{\mathscr{B}_{i}^{p}\right\}$ является $(k+p)$-ВР-сложным, откуда следует, что класс $\mathscr{B}^{p}$ является $(k+p)$-ВР-предельным.

Действительно, для произвольного графа $G$ справедливо равенство

$$
\chi\left(G \circ K_{p}\right)=\chi(G)+p .
$$

Таким образом, неравенство $\chi\left(G \circ K_{p}\right) \leqslant p+k$ выполняется тогда и только тогда, когда выполняется неравенство $\chi(G) \leqslant k$. Поэтому при любом $i$ класс $\mathscr{B}_{i}^{p}$ является $(k+p)$ ВР-сложным. 
Теперь докажем, что класс $\mathscr{B}^{p}$ является $(k+p)$-ВР-граничным. Предположим, что это не так. Тогда ввиду леммы 1 существует бесконечная монотонно убывающая последовательность $(k+p)$-сложных частей класса $\mathscr{L} p$, сходящаяся к собственному подклассу класса $\mathscr{B}^{p}$. Назовем эту последовательность первой. Сформируем новую последовательность, которую назовем второй. Для каждого члена первой последовательности выберем все графы $H$, представимые в виде $G \circ K_{p}$. Понятно, что такое разложение графа $H$ единственно (с точностью до изоморфизма) и что формирование графа $G$ осуществимо за полиномиальное время. Очевидно, что все исключенные графы заведомо имеют хроматическое число, не превосходящее $k+p$. Поэтому порожденные подграфы графов $G$ составляют $k$-ВР-сложный класс, являющийся членом второй последовательности. Очевидно, что вторая последовательность сходится к собственному подклассу класса Я. Получаем противоречие с его $k$-ВР-граничностью. Таким образом, $\mathscr{B}^{p} \in \mathcal{B}_{k+p}$. Теорема 1 доказана.

Теорема 1 позволяет установить континуальность множества $\mathcal{B}_{k}$ при любом $k \geqslant 4$. Для $k=3$ это сделано в работе [2], где указано континуальное семейство $\mathcal{F} 3$-ВР-граничных подклассов класса $\operatorname{Deg}(4) \cap \operatorname{Free}\left(\left\{K_{5}\right\}\right)$. Данный класс является конечно определенным, и из известной теоремы Брукса следует, что

$$
\operatorname{Deg}(4) \cap \operatorname{Free}\left(\left\{K_{5}\right\}\right) \subseteq \operatorname{Colour}(4) .
$$

Поэтому из леммы 2 следует включение $\mathcal{F} \subseteq \mathcal{B}$, а из теоремы 1 следует, что при любом $k \geqslant 4$ множество $\mathcal{F}$ порождает в $\mathcal{B}_{k}$ континуальное подмножество. Таким образом, при любом $k \geqslant 4$ семейство $\mathcal{F}$ порождает континуальное множество $k$-ВР-граничных классов, каждый из которых не является ХЧ-граничным.

Вместе с тем, интересно было бы выяснить, существуют ли ХЧ-граничные классы, не являющиеся $k$-ВР-граничными ни при каком $k$. Ответ на этот вопрос дает следующее утверждение.

Теорема 2. Класс со(D) принадлежит $\mathcal{B} \backslash \bigcup_{i=3}^{\infty} \mathcal{B}_{i}$.

Доказательство. Класс со(Ф) является ХЧ-граничным [5]. Покажем, что он не является $s$-ВР-граничным ни при каком $s$. Действительно, для любого $s$ граф $K_{s+1}$ принадлежит классу со(D) и не принадлежит классу Colour $(s)$. Отсюда и из леммы 3 будет следовать, что $\operatorname{co}(\mathscr{D}) \notin \mathcal{B}_{s}$ для любого $s$. Теорема 2 доказана.

\section{Список литературы}

1. Малышев Д. С., О бесконечности множества граничных классов в задаче о реберной 3-раскраске. Дискретный анализ и исследование операџий (2009) 16, №1, 37-43.

2. Малышев Д. С., Континуальные множества граничных классов графов для задач о раскраске. Дискретный анализ и исследование операций (2009) 16, №5, 41-51.

3. Alekseev V. E., On easy and hard hereditary classes of graphs with respect to the independent set problem. Discrete Appl. Math. (2004) 132, 17-26.

4. Алексеев В. Е., Малышев Д. С., Критерий граничности и его применения. Дискретный анализ $u$ исследование операџий (2008) 15, №6, 3-10.

5. Малышев Д. С., Исследование грании эффективной разрешимости в семействе наследственных классов графов, Дисс. на соискание уч. степени канд. физ.-мат. наук, Нижний Новгород, 2009.

Статья поступила 19.01.2010. 